\title{
Nonconforming Mixed Finite Element Method for Nonlinear Hyperbolic Equations
}

\author{
Haihong Wang ${ }^{1}$, Cheng Guo ${ }^{2}$ \\ ${ }^{1}$ Department of Mathematical and Information Scientific, Henan University of Economics and Low, Zhengzhou, China \\ ${ }^{2}$ Department of Mathematics, Zhengzhou Normal University, Zhengzhou, China \\ Email: \{waih777,gc_scv\}@163.com
}

Received December 22, 2011; revised February 13, 2012; accepted February 21, 2012

\begin{abstract}
A nonconforming mixed finite element method for nonlinear hyperbolic equations is discussed. Existence and uniqueness of the solution to the discrete problem are proved. Priori estimates of optimal order are derived for both the displacement and the stress.
\end{abstract}

Keywords: Nonconforming Mixed Finite Element; Hyperbolic Equations; Semi-Discrete Scheme; Error Estimates

\section{Introduction}

In this paper, we discuss a nonconforming mixed finite element method for the following nonlinear hyperbolic initial and boundary value problem.

$$
\left\{\begin{array}{l}
b(u) u_{t t}-\nabla \cdot(a(u) \nabla u)=f(u),(x, y) \in \Omega, t \in(0, T] \\
u(X, t)=-g(X, t),(x, y) \in \partial \Omega, t \in(0, T] \\
u(x, y, 0)=u_{0}(x, y), u_{t}(x, y, 0)=u_{1}(x, y),(x, y) \in \Omega,
\end{array}\right.
$$

In order to describe the results briefly, we suppose that Equation (1) satisfy following assumptions on the data:

1) $a(u)$ and $b(u)$ are smooth and there exist constants $c_{o}, c_{1}, a_{0}$ and $a_{1}$ satisfying

$$
0<c_{0} \leq b(u) \leq c_{1}, 0<a_{0} \leq a(u) \leq a_{1} .
$$

2) $f(u), a(u), g(X, t), u_{0}(X)$ and $u_{1}(X)$ are sufficiently smooth functions with bounded derivatives.

There have been many very extensive studies about this kind of hyperbolic equations. For example, [1-3] studied the linear situations and gave error estimates under semi-discrete and fully-discrete schemes by standard Galerkin methods. [4,5] considered the mixed finite element methods for linear hyperbolic equations and obtained $L^{2}$ prior estimates about continuous time. In addition, [6] analyzed the mixed finite element methods for second order nonlinear hyperbolic equations. But all the above investigations are mainly about conforming situations and projections are indispensable. As we know, the nonconforming finite element methods arise because of the demands for reducing the calculation cost. [7] has pointed that the nonconforming finite element methods with degree of freedom defined on the element edges or element itself are appropriate for each degree of freedom belong to at most elements.

In the present work, we focus on the nonconforming mixed finite element approximation scheme for nonlinear hyperbolic equations. Firstly, we introduce the corresponding space and the interpolation operators. Secondly, Existence and uniqueness of the solutions to the discrete problem are proved. Finally, Priori estimates of optimal order are derived for both the displacement and the stress.

Throughout this paper, $C$ denotes a general positive constant which is independent of $h=\max \left\{h_{K}\right\}$, and $h_{K}$ is the diameter of the finite element $K$.

\section{Construction of the Elements}

Let $J_{h}$ be the a rectangular subdivision of $\Omega \subset R^{2}$, and $\bar{\Omega}=\bigcup_{K \in J_{h}} K$ satisfy the regular condition. For every $K$, let $\left(x_{K}, y_{K}\right)$ be the barycenter, the length of edges parallel to $\mathrm{x}$-axis and $\mathrm{y}$-axis by $2 h_{x}$ and $2 h_{y}$. Then there exists an affine mapping

$$
F_{K}: \hat{K} \rightarrow K, x=x_{K}+h_{x} \hat{x}, y=y_{K}+h_{y} \hat{y},
$$

where $\hat{K}=[0,1] \times[0,1]$ is the reference element in $(\hat{x}, \hat{y})$ plane and $l_{i}$ is the edges. We define the finite element $\left(\hat{K}, \hat{P}^{i}, \hat{\Sigma}^{i}\right)(i=1,2,3)$ on $\hat{K}$ as

$$
\begin{aligned}
& \hat{\Sigma}^{1}=\left\{\frac{1}{|\hat{K}|} \int_{\hat{K}} \hat{\lambda} \mathrm{d} \hat{x} \mathrm{~d} \hat{y}\right\}, \hat{P}^{1}=\operatorname{span}\{1\}, \\
& \hat{\Sigma}^{2}=\left\{\hat{q}_{1}, \hat{q}_{2}, \hat{q}_{3}, \hat{q}_{4}\right\}, \hat{P}^{2}=\operatorname{span}\left\{1, \hat{x}, \hat{y}, \hat{y}^{2}\right\},
\end{aligned}
$$




$$
\begin{aligned}
& \hat{\Sigma}^{3}=\left\{\hat{p}_{1}, \hat{p}_{2}, \hat{p}_{3}, \hat{p}_{4}\right\}, \hat{P}^{2}=\operatorname{span}\left\{1, \hat{x}, \hat{y}, \hat{x}^{2}\right\}, \\
& \hat{q}_{i}=\frac{1}{\left|\hat{l_{i}}\right|} \int_{\hat{l}_{i}} \hat{q} \mathrm{~d} \hat{s}, \hat{p}_{i}=\frac{1}{\left|\hat{l}_{i}\right|} \int_{\hat{l}_{i}} \hat{p} \mathrm{~d} \hat{s}, i=1,2,3,4 .
\end{aligned}
$$

The interpolation functions defined above are properly and can be expressed as:

$$
\begin{aligned}
& \hat{I}^{1}=\frac{1}{|\hat{K}|} \int_{\hat{K}} \hat{\lambda} \mathrm{d} \hat{x} \mathrm{~d} \hat{y}, \\
& \hat{I}^{j} \hat{v}= 0.25\left(-\hat{v}_{1}-\hat{v}_{2}+3 \hat{v}_{3}+3 \hat{v}_{4}\right)+0.5\left(-\hat{v}_{2}+\hat{v}_{4}\right) \hat{x} \\
&+0.5\left(-\hat{v}_{2}+\hat{v}_{4}\right) \hat{x}+0.5\left(-\hat{v}_{1}+\hat{v}_{3}\right) \hat{y} \\
&+0.75\left(\hat{v}_{1}-\hat{v}_{2}+\hat{v}_{3}-\hat{v}_{4}\right) \hat{y}^{2}
\end{aligned}
$$

$v=p$ or $q, j=2,3$. And For every $\hat{p}, \hat{v} \in H^{1}(\Omega)$, the associated finite element spaces as

$$
\begin{aligned}
V_{h} & =\left\{v ;\left.v\right|_{K}=\hat{v} \circ F_{K}^{-1}, \hat{v} \in \hat{P}^{1}\right\}, \\
W_{h} & =\left\{w=\left(w_{1}, w_{2}\right) ;\left.w\right|_{K}=\left(\hat{w}_{1} \circ \hat{F}_{K}^{-1}, \hat{w}_{1} \circ \hat{F}_{K}^{-1}\right)\right. \\
& \left.\in \hat{P}^{2} \times \hat{P}^{3}, \int_{F}[w] \mathrm{d} s=0, F \subset \partial \Omega\right\},
\end{aligned}
$$

where $[w]$ denotes the jump of $w$ across the boundary $F$, and $[w]=w$, if $F \subset \partial \Omega . \forall v \in L^{2}(\Omega)$,

$\forall w \in\left(H^{1}(\Omega)\right)^{2}$, the interpolation operators:

$$
\begin{aligned}
& \left.I_{h}^{1}\right|_{K}=I_{K}^{1}, I_{K}^{1} v=\left(\hat{I}^{1} \hat{v}\right) \circ F_{K}^{-1}, . \\
& \left.I_{h}^{2}\right|_{K}=I_{K}^{2}, I_{K}^{2} v=\left(\left(\hat{I}^{2} \hat{v}\right) \circ F_{K}^{-1},\left(\hat{I}^{2} \hat{w}_{2}\right) \circ F_{K}^{-1}\right) .
\end{aligned}
$$

\section{Main Results in Semi-Discrete Scheme}

In this section, we will give the main results in this paper, including the existence and uniqueness of the solution to the discrete problem and priori estimates of optimal order.

Firstly, we introduce

$$
p=-a(u) \nabla u, \alpha(u)=1 / a(u),
$$

and rewrite the Equation (1) as a system:

$$
b(u) u_{t t}+\nabla \cdot p=f(u), \alpha(u) p+\nabla u=0 .
$$

Secondly, for our subsequent use, we employ the classical Sobolev space $W^{m, p}(\Omega)$ with norm $\|\cdot\|_{m . p}$. When $p=2$, we simply write $\|\cdot\|_{m . p}$ as $\|\cdot\|_{m}$. Furthermore, we denote the natural inner production in $L^{2}(\Omega)$ by $(\cdot, \cdot)$ and the norm by $\|\cdot\|$, and let

$$
\begin{gathered}
H_{0}^{1}(\Omega)=\left\{v \in H^{1}(\Omega):\left.v\right|_{\partial \Omega}=0\right\}, \\
H(\operatorname{div} ; \Omega)=\left\{w \in\left(L^{2}(\Omega)\right)^{2}, \nabla \cdot w \in L^{2}(\Omega)\right\} .
\end{gathered}
$$

Thus the corresponding weak formulation of Equation (1) is to find a pair of $\{u, p\}$, such that $[0, T] \rightarrow H_{0}^{1}(\Omega) \times H(\operatorname{div} ; \Omega)$ satisfying

$$
\left\{\begin{array}{l}
\left(b(u) u_{t t}, v\right)+(\nabla \cdot p, v)=(f(u), v), \forall v \in L^{2}(\Omega) \\
(\alpha(u) p, w)-(u, \nabla \cdot w)=\langle g, w \cdot n\rangle, \forall w \in H(\operatorname{div} ; \Omega) \\
u(x, 0)=u_{0}(x), u_{t}(x, 0)=u_{1}(x), x \in \Omega,
\end{array}\right.
$$

where $\langle g, w \cdot n\rangle=\int_{\partial \Omega} g w \cdot n \mathrm{~d} s$.

The semi-discrete mixed finite element procedure is determined: $\left\{u_{h}, q_{h}\right\}:[0, T] \rightarrow V_{h} \times W_{h}$, such that

$$
\left\{\begin{array}{l}
\left(b\left(u_{h}\right) u_{h t t}, v_{h}\right)+\left(\nabla \cdot p_{h}, v_{h}\right)=\left(f\left(u_{h}\right), v_{h}\right), \forall v_{h} \in V_{h} \\
\left(\alpha\left(u_{h}\right) p_{h}, w_{h}\right)-\left(u_{h}, \nabla \cdot w_{h}\right)=\left\langle g, w_{h} \cdot n\right\rangle, \forall w_{h} \in W_{h} \\
u_{h}(x, 0)=I_{h}^{1} u_{0}(x), u_{h t}(x, 0)=I_{h}^{1} u_{1}(x), x \in \Omega,
\end{array}\right.
$$

where $\left\langle g, w_{h} \cdot n\right\rangle=\sum_{K} \int_{\partial K} g w_{h} \cdot n \mathrm{~d} s$. We define that

$$
\begin{gathered}
\left|v_{h}\right|_{h}=\left(\sum_{K}\left|v_{h}\right|_{1, K}^{2}\right)^{1 / 2}, \forall v_{h} \in V_{h}, \\
\left\|w_{h}\right\|_{h}=\sum_{K}\left(\left\|w_{h}\right\|^{2}+\left\|\nabla \cdot w_{h}\right\|^{2}\right)^{1 / 2}, w_{h} \in W_{h} .
\end{gathered}
$$

It can be seen that $\mid \varphi_{h}$ and $\|\cdot\|_{h}$ are the norms for $V_{h}$ and $W_{h}$, respectively.

Theorem 1. The above problem (3) has a unique solution.

Proof: Let $\left\{\psi_{i}\right\}_{i=1}^{r_{1}}$ and $\left\{\phi_{i}\right\}_{i=1}^{r_{2}}$ are bases of $V_{h}$ and $W_{h}$, which satisfy

$$
u_{h}=\sum_{i=1}^{r_{1}} \phi_{i}(t) \psi_{i}, p_{h}=\sum_{j=1}^{r_{2}} \lambda_{j}(t) \phi_{j}
$$

Then semi-discrete scheme can be rewritten as: Find $\phi_{i}(t)\left(i=1,2, \cdots, r_{1}\right)$ and $\lambda_{j}(t)\left(j=1,2, \cdots, r_{2}\right)$, such that for every $t \in\left(0, t_{1}\right)$ satisfy

$$
\left\{\begin{array}{l}
A \frac{\mathrm{d}^{2}(\phi(t))}{\mathrm{d} t^{2}}+B \lambda(t)=D, \\
E \lambda(t)+F \phi(t)=G,
\end{array}\right.
$$

here

$$
\begin{aligned}
& A=\left(b\left(\sum_{i=1}^{r_{1}} \phi_{i}(t) \psi_{i}\right) \psi_{i}, \psi_{j}\right)_{r_{1} \times r_{2}}, B=\left(\left(\nabla \cdot \phi_{i}, \phi_{j}\right)\right)_{r_{1} \times r_{2}}, \\
& D=\left(\left(f\left(\sum_{i=1}^{r_{1}} \phi_{i}(t) \psi_{i}\right) \psi_{i}, \psi_{j}\right)\right)_{1 \times r_{1}}, \quad F=\left(\left(\left\langle g, \phi_{i} \cdot n\right\rangle\right)\right)_{1 \times r_{1}}^{\prime} \\
& G=\left(\left(\lambda\left(\sum_{j=1}^{r_{2}} \lambda_{j}(t) \phi_{j}\right) \phi_{i}, \phi_{j}\right)\right)_{r_{2} \times r_{2}},
\end{aligned}
$$

By the definition of the approximation spaces, we 
know that $E$ is reversible and $\lambda(t)=E^{-1}(G-F \psi(t))$. Thus there holds that

$$
A \frac{\mathrm{d}^{2}(\varphi(t))}{\mathrm{d} t^{2}}-B E^{-1} F \varphi(t)=D-B E^{-1} G .
$$

Since $A, B E^{-1} F$ and $D-B E^{-1} G$ are Lipschitz continuous, it has a unique solution according to the theory of differential equations [8].

Lemma 1. For $\forall u \in H^{1}(\Omega), \quad p \in H(\operatorname{div} ; \Omega), v \in V_{h}$, there hold that

$$
\begin{gathered}
\operatorname{div} V_{h}=W_{h}, \\
\left(u-I_{h}^{1} u, \psi\right)=0,\left(\nabla \cdot\left(p-I_{h}^{2} p\right), v\right)=0,
\end{gathered}
$$

Proof: Firstly, by the interpolation condition and definition, it is easy to see that $\operatorname{div} V_{h}=W_{h}$ and $\left(u-I_{h}^{1} u, \psi\right)=0$. Secondly, for every $v \in V_{h}, v$ is a constant, by application of Green's formula and the interpo- lation definition yields that

$$
\begin{aligned}
& \left(\nabla \cdot\left(p-I_{h}^{2} p\right), v\right)=\sum_{K} \int_{K} \nabla \cdot\left(p-I_{K}^{2} p\right) \psi \mathrm{d} x \mathrm{~d} y \\
& =\sum_{K} \int_{K}\left(p-I_{K}^{2} p\right) \nabla \psi \mathrm{d} x \mathrm{~d} y+\sum_{K} \int_{\partial K}\left(p-I_{K}^{2} p\right) \cdot n \psi \mathrm{d} s \\
& =\sum_{K} \psi \int_{\partial K}\left(p-I_{K}^{2} p\right) \cdot n \mathrm{~d} s=0 .
\end{aligned}
$$

Thus, we complete the proof of Lemma 1.

Lemma 2. [9] For $u_{t} \in H^{2}(\Omega)$, there hold that

$$
\sum_{K} \int_{\partial K} u_{t}(\psi \cdot n) \mathrm{d} s \leq C h|u|_{2}\|\psi\|, \quad \forall \psi \in W_{h}
$$

Now we give the main result of this paper.

Theorem 2. Let $\{u, p\}$ and $\left\{u_{h}, p_{h}\right\}$ be the solutions of Equations (2) and (3), respectively. For $u, u_{t} \in H^{1}(\Omega), u_{t} \in H^{2}(\Omega), p \in\left(H^{1}(\Omega)\right)^{2}$, there hold that

$$
\left\|u-u_{h}\right\|+\left\|u_{t}-u_{h t}\right\|+\left\|p-p_{h}\right\| \leq C h\left[|u|_{1}+\left|u_{t}\right|_{1}+|p|_{1}+\left(\int_{0}^{t}\left(|u|_{1}^{2}+\left|u_{t}\right|_{1}^{2}+\left|u_{t t}\right|_{1}^{2}+\left|u_{t}\right|_{2}^{2}+|p|_{1}^{2}\right) \mathrm{d} \tau\right)^{1 / 2}\right] .
$$

Proof: Let $u-u_{h}=u-I_{h}^{1} u+I_{h}^{1} u-u_{h}=\eta+\xi$,

$p-p_{h}=p-I_{h}^{2} p+I_{h}^{2} p-p_{h}=\sigma+\theta$.

It is easy to see that $v_{h} \in V_{h}$ and $w_{h} \in W_{h}$ satisfy the following error equations

$$
\begin{aligned}
& \left(b\left(u_{h}\right) \xi_{t t}, v_{h}\right)+\left(\nabla \cdot \theta, v_{h}\right)=\left(b\left(u_{h}\right) \theta_{t t}, v_{h}\right) \\
& +\left(f\left(u_{h}\right)-f(u), v_{h}\right)+\left(\left(b(u)-b\left(u_{h}\right)\right) u_{t t}, v_{h}\right)
\end{aligned}
$$

and

$$
\begin{aligned}
& \left(\alpha\left(u_{h}\right) \theta, w_{h}\right)-\left(\xi, \nabla \cdot w_{h}\right)=\left(\alpha\left(u_{h}\right) \sigma, w_{h}\right) \\
& +\left(\left(\alpha(u)-\alpha\left(u_{h}\right)\right) p, w_{h}\right)+\sum_{K} \int_{\partial K} u w_{h} \cdot n d s .
\end{aligned}
$$

Using derivation about time $t$ of Equation (4), combining Equation (5), we obtain

$$
\begin{aligned}
& \left(b\left(u_{h}\right) \xi_{t t}, v_{h}\right)+\left(\alpha\left(u_{h}\right) \theta_{t}, w_{h}\right)=\left(b\left(u_{h}\right) \eta_{t t}, v_{h}\right)+\left(f\left(u_{h}\right)-f(u), v_{h}\right)+\left(\left(b(u)-b\left(u_{h}\right)\right) u_{t t}, v_{h}\right) \\
& +\left(\left(\left(\alpha(u)-\alpha\left(u_{h}\right)\right) p\right)_{t}, w_{h}\right)+\left(\alpha\left(u_{h}\right) \sigma, w_{h}\right)+\left(\left(\alpha\left(u_{h}\right)\right)_{t}(\sigma-\theta), w_{h}\right)+\sum_{K} \int_{\partial K} u w_{h} \cdot n d s
\end{aligned}
$$

Choosing $v_{h}=\xi_{t}$ and $w_{h}=\theta$ in Equation (6), and integrating from 0 to $t$, we have

$$
\begin{aligned}
& \int_{0}^{t}\left(b\left(u_{h}\right) \xi_{t t}, \xi_{t}\right)+\left(\alpha\left(u_{h}\right) \theta_{t}, \theta\right) \mathrm{d} \tau=\int_{0}^{t}\left[\left(b\left(u_{h}\right) \eta_{t t}, \xi_{t}\right)+\left(\left(\alpha\left(u_{h}\right)\right)_{t}(\sigma-\theta), \theta\right)+\left(\alpha\left(u_{h}\right) \sigma, \theta\right)\right] \mathrm{d} \tau \\
& +\int_{0}^{t}\left(f\left(u_{h}\right)-f(u), \xi_{t}\right)+\int_{0}^{t}\left(\left(b(u)-b\left(u_{h}\right)\right) u_{t t}, \xi_{t}\right) \mathrm{d} \tau+\int_{0}^{t}\left(\left(\left(\alpha(u)-\alpha\left(u_{h}\right)\right) p\right)_{t}, \theta\right) \mathrm{d} \tau+\int_{0}^{t} \sum_{K} \int_{\partial K} u \theta \cdot n \mathrm{~d} s \mathrm{~d} \tau
\end{aligned}
$$

We will give the analysis result of Equation (7) in detail. Firstly, by the initial condition, it is followed that

$$
\begin{aligned}
& \int_{0}^{t}\left(b\left(u_{h}\right) \xi_{t t}, \xi_{t}\right)+\left(\alpha\left(u_{h}\right) \theta_{t}, \theta\right) \mathrm{d} \tau \\
& =\frac{1}{2} \int_{0}^{t}\left[\frac{\mathrm{d}}{\mathrm{d} \tau}\left(\left(b\left(u_{h}\right) \xi_{\tau}, \xi_{\tau}\right)\right)-\left(\left(\left(b\left(u_{h}\right)\right)_{\tau} \xi_{\tau}, \xi_{\tau}\right)+\frac{\mathrm{d}}{\mathrm{d} \tau}\left(\left(\alpha\left(u_{h}\right) \theta, \theta\right)-\left(\left(\alpha\left(u_{h}\right)\right)_{\tau} \theta, \theta\right)\right)\right] \mathrm{d} \tau\right. \\
& \geq C\left(\left\|\xi_{t}\right\|^{2}+\frac{1}{a_{1}}\|\theta\|^{2}-\int_{0}^{t}\left(\left\|\xi_{t}(\tau)\right\|^{2}+\|\theta(\tau)\|^{2}\right) \mathrm{d} \tau\right)
\end{aligned}
$$

Secondly, by Cauchy-Schwartz's inequality and Young's inequality, we obtain

$$
\int_{0}^{t}\left(b\left(u_{h}\right) \eta_{t t}, \xi_{t}\right)+\left(\left(\alpha\left(u_{h}\right)\right)_{t}(\sigma-\theta), \theta\right)+\left(\alpha\left(u_{h}\right) \sigma, \theta\right) \mathrm{d} \tau \leq C \int_{0}^{t}\left(\left\|\eta_{t t}(\tau)\right\|^{2}+\left\|\xi_{t}(\tau)\right\|^{2}+\|\sigma(\tau)\|^{2}+\|\theta(\tau)\|^{2}\right) \mathrm{d} \tau
$$

Similarly, by the initial condition of $f(u), b(u)$ and $a(u)$, we use Young's inequality to get 


$$
\left\{\begin{array}{l}
\int_{0}^{t}\left(f\left(u_{h}\right)-f(u), \xi_{t}\right) \mathrm{d} \tau \leq C \int_{0}^{t}\left(\|\eta(\tau)\|^{2}+\|\xi(\tau)\|^{2}+\left\|\xi_{t}(\tau)\right\|^{2}\right) \mathrm{d} \tau, \\
\int_{0}^{t}\left(\left(b(u)-b\left(u_{h}\right) u_{t t}, \xi_{t}\right) \mathrm{d} \tau \leq C \int_{0}^{t}\left(\|\eta(\tau)\|^{2}+\|\xi(\tau)\|^{2}+\left\|\xi_{t}(\tau)\right\|^{2}\right) \mathrm{d} \tau\right. \\
\int_{0}^{t}\left(\left(\left(\alpha(u)-\alpha\left(u_{h}\right)\right) p\right)_{t}, \theta\right) d \tau \leq C \int_{0}^{t}\left(\left\|\eta_{t}(\tau)\right\|^{2}+\|\eta(\tau)\|^{2}+\|\xi(\tau)\|^{2}+\left\|\xi_{t}(\tau)\right\|^{2}+\|\theta(\tau)\|^{2}\right) \mathrm{d} \tau
\end{array}\right.
$$

Substituting the above estimates, and applying Lemma 2, we get

$$
\left\|\xi_{t}\right\|^{2}+\|\theta\|^{2} \leq C \int_{0}^{t}\left(\left\|\eta_{t}(\tau)\right\|^{2}+\|\eta(\tau)\|^{2}+\|\xi(\tau)\|^{2}+\left\|\xi_{t}(\tau)\right\|^{2}+\|\theta(\tau)\|^{2}+\left\|\eta_{t t}(\tau)\right\|^{2}+\|\sigma(\tau)\|^{2}+h^{2}\left|u_{t}(\tau)\right|_{2}^{2}\right) \mathrm{d} \tau
$$

Then adding $\|\xi\|^{2}$ at both sides Equation (11), and noticing that $\|\xi\|^{2} \leq C \int_{0}^{t}\left\|\xi_{t}(\tau)\right\|^{2} \mathrm{~d} \tau$, we obtain that

$$
\|\xi\|^{2}+\left\|\xi_{t}\right\|^{2}+\|\theta\|^{2} \leq C h^{2} \int_{0}^{t}\left(|u(\tau)|_{1}^{2}+\left|u_{t}(\tau)\right|_{1}^{2}+\left|u_{t t}(\tau)\right|_{1}^{2}+\left|u_{t}(\tau)\right|_{2}^{2}+|p(\tau)|_{1}^{2}+\|\xi(\tau)\|^{2}+\left\|\xi_{t}(\tau)\right\|^{2}+\|\theta(\tau)\|^{2}\right) \mathrm{d} \tau
$$

Further, using Gronwall’s inequality to yield

$$
\|\xi\|+\left\|\xi_{t}\right\|+\|\theta\| \leq C h\left[\int_{0}^{t}\left(|u(\tau)|_{1}^{2}+\left|u_{t}(\tau)\right|_{1}^{2}+\left|u_{t t}(\tau)\right|_{1}^{2}+\left|u_{t}(\tau)\right|_{2}^{2}+|p(\tau)|_{1}^{2}\right) \mathrm{d} \tau\right]^{1 / 2}
$$

By the interpolation theory (see [10]), we have

$$
\|\eta\|+\left\|\eta_{t}\right\|+\|\sigma\| \leq C h\left(|u|_{1}+\left|u_{t}\right|_{1}+|p|_{1}\right) .
$$

Finally, by the triangle inequality, we complete the proof.

\section{REFERENCES}

[1] T. Dupont, " $L^{2}$-Estimates for Galerkin Methods for Second Order Hyperbolic Equations," SIAM Journal on $\mathrm{Nu}$ merical Analysis, Vol. 10, No. 1, 1973, pp. 880-889. doi: $10.1137 / 0710073$

[2] T. Oden and J. Reddy, "An Introduction to the Mathematical Theory of Finite Elements,” Wiley Interscience, New York, 1976.

[3] G. A. Baker, "Error Estimates for Finite Element Methods for Second Hyperbolic Equations," SIAM Journal on Numerical Analysis, Vol. 13, No. 1, 1976, pp. 564-576. doi:10.1137/0713048

[4] J. J. Douglas, "Superconvergence in the Pressure in the Simulation of Miscible Displacement," SIAM Journal on Numerical Analysis, Vol. 22, No. 1, 1985, pp. 962-969. doi:10.1137/0722058
[5] L. C. Cowsar, T. F. Dupont and M. T. Wheeler, “A Priori Estimates for Mixed Finite Element Approximations of Second-Order Hyperbolic Equations with Absorbing Boundary Conditions," Computer Methods in Applied Mechanic and Engineering, Vol. 33, No. 1, 1996, pp. 492504.

[6] Y. P. Chen and Y. Q. Huang, "Mixed Finite Element Method for Nonlinear Hyperbolic Equations,” Numerical Mathematics A Journal of Chinese University, Vol. 1, 2000, pp. 63-69.

[7] S. Martin and T. Lutz, "The Streamline-Diffusion Method for Nonconforming $Q^{\text {rot }}$ Elements on Rectangular Tensor-Product,” IMA Journal Numerical Analysis, Vol. 21, No. 1, 2001, pp: 123-142. doi:10.1093/imanum/21.1.123

[8] J. K. Hale, “Ordinary Differential Equations,” WilleyInterscience, New York, 1969.

[9] D. Y. Shi and H. H. Wang, "Nonconforming $H^{1}$-Galerkin Mixed FEM for Sobolev Equations on Anisotropic Meshes,” Acta Mathematicae Applicatae Sininica, Vol. 25, No. 2B, 2009, pp. 335-344. doi:10.1007/s10255-007-7065-y

[10] P. G. Ciarlet, "The Finite Element Method for Elliptic Problem,” North-Holland, Amsterdam, 1978. 